\title{
New roles for TRPM7
}

TRPM7 (transient receptor potential melastatin related 7) is an ion channel and protein kinase that has been suggested to be involved in $\mathrm{Mg}^{2+}$ homeostasis. Now, Clapham and colleagues show that TRPM7 is essential for embryonic development and thymopoiesis but not for $\mathrm{Mg}^{2+}$ homeostasis. The expression of TRPM7 by thymocytes is required for the transition of thymocytes from the double negative (DN) stage of development and for the maintenance of medullary thymic epithelial cells (mTECs).

To examine the role of TRPM7 in T-cell development, the authors generated mice with a conditional deletion of Trpm 7 in T cells. They found that TRPM7 does not mediate $\mathrm{Mg}^{2+}$ influx in freshly isolated T cells and is not essential for cellular $\mathrm{Mg}^{2+}$ homeostasis. In addition, mature
Trpm7-deficient T cells survived and populated the intestine and lymph nodes, and showed normal migratory patterns in the periphery. However, the thymus of these mice had structural abnormalities.

The boundary between the cortex and medulla of the thymus was not visible and, in contrast to wild-type mice in which $\mathrm{CD}^{+}$thymocytes are confined to the medulla, Trpm7-deficient thymocytes were distributed throughout the thymus. These mice also had a reduced total number of thymocytes, but had a higher percentage and number of thymocytes at the DN stage of development compared with control mice. Further analysis showed that a proportion of $\operatorname{Trpm} 7$-deficient thymocytes exhibited a block during the DN3 to DN4 transition stage.

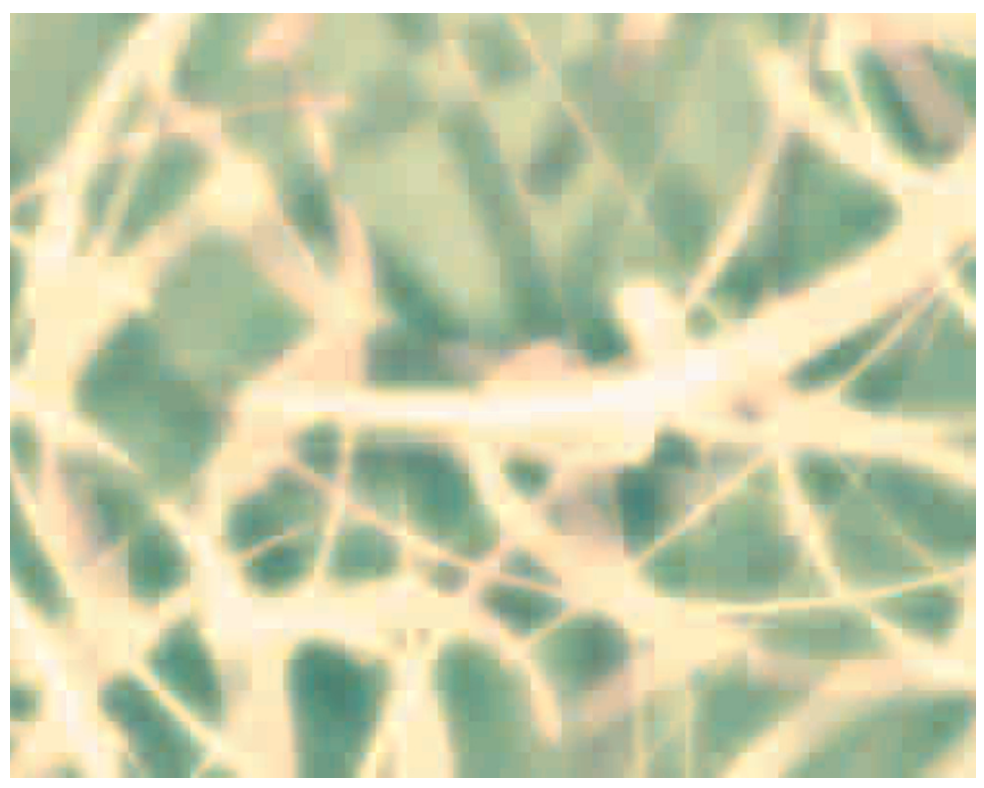

Immunofluorescence staining of thymus sections from 4- and 12-week-old Trpm7-conditionalknockout mice showed a progressive loss of mTECs but not cortical TECs. Analysis of the expression of 82 growth factors in Trpm7-deficient thymocytes identified 5 molecules, including fibroblast growth factor 7 (FGF7) and midkine, that were expressed at significantly lower levels than in control cells. FGF7 and midkine induce the activation of signal transducer and activator of transcription 3 (STAT3)-mediated responses in mTECs, which are crucial for the maintenance of thymic architecture. Indeed, STAT3, which is autoregulated, was shown to be expressed by mTECs in wild-type mice, but its expression was progressively lost in Trpm7-conditional knockout mice.

So, the data show that loss of Trpm 7 in thymocytes results in the aberrant development and distribution of thymocytes, a reduced expression of STAT3 in mTECs and a progressive loss of these cells. The authors suggest that reduced STAT3 expression could lead to the disrupted architecture of the thymus in Trpm7-conditional knockout mice. However, further studies are needed to identify the molecular mechanisms that cause the mislocalization of thymocytes and the block in thymocyte development.

Olive Leavy

ORIGINAL RESEARCH PAPER Jin, J. et al. Deletion of Trpm7 disrupts embryonic development and thymopoiesis without altering $\mathrm{Mg}^{2+}$ homeostasis. Science 322, 756-760 (2008) 